\title{
"EVALUACION DEL DESEMPEÑO DOCENTE EN LA PRÁCTICA CLINICA DE LA ASIGNATURA SALUD DEL ADULTO Y ANCIANO EN LA FACULTAD DE ENFERMERÍA DE LA UNIVERSIDAD NACIONAL SAN LUIS GONZAGA DE ICA, 2017"
}

Evaluation of teaching performance in the clinical practice of the course health of the adult and ancient in the Faculty of Nursing of the San Luis Gonzaga from Ica National University, 2017

\section{Rosa Herlinda Hernández Onofra ${ }^{1,3, a, c}$, Amanda María García Aquije ${ }^{3, a, c}$, Katherine Milagros Cornejo García ${ }^{2, b}$}

\footnotetext{
${ }^{1}$ Facultad de Enfermería de la Universidad Nacional San Luis Gonzaga de Ica

${ }^{2}$ Facultad de Medicina de la Universidad Nacional San Luis Gonzaga de Ica

${ }^{3}$ Hospital Augusto Hernández Mendoza - Essalud, Ica.

a Licenciada en Enfermería, ${ }^{b}$ Médico Cirujano, "Magíster en Salud Pública.
}

\section{RESUMEN}

El presente estudio tuvo por Objetivo: Evaluar el desempeño docente en los estudiantes de enfermería sobre la enseñanza en la práctica clínica de la asignatura Enfermería en Salud del Adulto y Anciano, 2017. Material y métodos: Estudio descriptivo, no experimental, de corte transversal, cuantitativo; la muestra constituida por 38 estudiantes de pregrado V Ciclo 2017-I. Como instrumento se utilizó un cuestionario de escala Likert modificada, con 22 enunciados sobre la evaluación del desempeño docente en la práctica clínica de la asignatura de Salud del Adulto y Anciano. Resultados: La evaluación del desempeño docente en la práctica de la asignatura de Salud del Adulto y Anciano es Excelente. $(p=0,000)$. Por área de prácticas, se encontró que la evaluación del desempeño docente en el área de medicina es de Bueno a Excelente. $(p=0,565)$; en el área de Cirugía es excelente. ( $p=0,001)$; en el área sala de operaciones es excelente. $(p=0,000)$; y en el área de Recuperación Postoperatorio es excelente $(p=0,000)$. Las características sociodemográficas evidenciadas en los estudiantes con mayor frecuencia son: Edad de 18 a 21 años $(86,8 \%)$, sexo femenino (78,9\%), y estado civil soltero (89,5\%). Conclusiones: La evaluación del desempeño docente en la práctica de la asignatura de Salud del Adulto y Anciano es Excelente.

Palabras clave: Evaluación desempeño docente, práctica clínica, estudiantes enfermería.

\section{SUMMARY}

The present study had as Objective: To evaluate the teaching performance of nursing students on teaching in the clinical practice of Nursing in Adult and Elderly Health, 2017. Material and methods: Descriptive, non-experimental, cross-sectional study quantitative; the sample constituted by 38 undergraduate students V Cycle 2017-I. As an instrument, a modified Likert scale questionnaire was used, with 22 statements on the evaluation of teaching performance in the clinical practice of the subject of Adult and Elderly Health. Results: The evaluation of the teaching performance in the practice of the subject of Health of the Adult and the Elderly is Excellent. $(p=0,000)$. By area of practice, it was found that the evaluation of teaching performance in the area of medicine is from Good to Excellent. $(p=0,565)$; in the area of Surgery is excellent. $(p=0,001)$; in the operating room area is excellent. $(p=0,000)$; and in the area of Postoperative Recovery is excellent $(p=0,000)$. The sociodemographic characteristics evidenced in the students with greater frequency are: Age from 18 to 21 years old $(86,8 \%)$, female sex $(78,9 \%)$, and single marital status $(89,5 \%)$. Conclusions: The evaluation of the teaching performance in the practice of the subject of Adult and Elderly Health is Excellent.

Key words: Teaching performance evaluation, clinical practice, nursing students. 


\section{INTRODUCCIÓN}

Si bien los procesos de evaluación docente realizados en las universidades, han tenido aspectos positivos, aun se advierten problemas, como la falta de claridad en los propósitos que orientan las acciones evaluativas, desarticuladas en los criterios de evaluación aplicados, además que es escasa la información que dé cuenta de cuales han sido los resultados obtenidos, sobre todo que cambios se han podido apreciar en la vida académica institucional (Rueda y Luna 2010) (1).

Muñoz, Ríos y Abalde (2002) afirman que el problema con la evaluación de la docencia surge generalmente porque tiene consecuencias escasas o nulas para la mejora de la calidad, tanto en la enseñanzaaprendizaje, como para el propio profesor, y por qué en la mayoría de los casos se lleva a cabo bajo un enfoque sancionador $y$ fiscalizador (2).

Otra problemática que surge en torno a la evaluación de la docencia, es que hasta ahora el medio más empleado para realizarla han sido los cuestionarios de opinión de los alumnos, tomándolos como la mejor fuente de información; no obstante en términos generales, se considera que pueden estar cargadas de subjetividad, e influidas por diversos factores, y sesgos. (Sánchez y Domínguez 2008) (3).

Al docente universitario actual se le exige un reciclaje continuo de sus conocimientos científicos, la capacidad de generar y publicar sus propios hallazgos, así como la capacidad de adaptarse al medio tecnológico y social, y ser además un docente portador de un amplio abanico de herramientas pedagógicas que le permitan transmitir todo ese conocimiento al alumnado, teniendo en cuenta el rol del estudiante activo en su propio aprendizaje (4).

El concepto de evaluación del desempeño docente en el caso de la educación superior, debe estar asociado a la toma de decisiones y a la implementación de los cambios para superar las debilidades detectadas. Por tanto, bajo este supuesto no basta con determinar los problemas y dificultades en la docencia, sino que también es necesario asociar las propuestas de acción para el mejoramiento, la definición y seguimiento de los mecanismos considerados para elevar los niveles de aprendizaje de los estudiantes, en el marco de las nuevas tendencias que en la actualidad se están dando en la educación superior (5).

La evaluación del desempeño es el proceso mediante el cual se estima el rendimiento global de un docente, constituye una función que, de una u otra forma, suele efectuarse en toda universidad. Para Colina Z. (2008), es un proceso sistemático de obtención de datos válidos y fiables, mide el efecto formativo y educativo del docente en los estudiantes (6).

La evaluación del desempeño requiere también disponer de mediciones del desempeño, que son los sistemas de calificación de cada labor, la evaluación puede darse a través de la observación del desempeño que puede ser directa o indirecta. La observación directa se da cuando quien califica el desempeño lo ve en persona, la observación indirecta ocurre cuando el evaluador debe basarse en otros elementos. También puede ser objetiva y subjetiva: Objetiva es la que otras personas pueden verificar; se basa en aspectos como por ejemplo, el número de alumnos evaluados, el número de evaluaciones aplicadas; es decir, se puede expresar en forma matemática; las mediciones subjetivas son las calificaciones no verificables, que pueden considerarse opiniones del evaluador, por ejemplo; la simpatía (6).Señalándose como objetivo evaluar el desempeño docente en los estudiantes de enfermería sobre la enseñanza en la práctica clínica de la asignatura Enfermería en Salud del Adulto y Anciano.

\section{MATERIAL Y MÉTODOS}

El presente estudio se llevó a cabo en la Facultad de Enfermería de la Universidad Nacional San Luis Gonzaga de Ica, cuyas instalaciones se encuentran ubicadas en la Ciudad Universitaria, Av. Los maestros $\mathrm{S} / \mathrm{N}$ de la ciudad de Ica, Perú. La población fue conformada por los alumnos del V Ciclo de la 
Facultad de enfermería de la Universidad Nacional San Luis Gonzaga de Ica. La muestra estuvo conformada por 38 estudiantes de pregrado, con matrícula del año 2017-I. El estudio de investigación por la naturaleza de su problema es de tipo cuantitativo, de corte transversal ya que los datos fueron obtenidos en un determinado periodo de tiempo y de nivel aplicativo ya que los resultados permitieron mejorar la calidad de la práctica clínica de los estudiantes de enfermería de la Universidad Nacional San Luis Gonzaga de Ica. El instrumento una escala de Likert modificada, el instrumento se ha diseñado en forma estructurada de acuerdo a los indicadores que constan de las siguientes partes:

a) Presentación, introducción (donde se da a conocer los estudiantes la finalidad del estudio, datos generales, las instrucciones

b) El contenido propiamente dicho donde se incluyeron 22 enunciados entre positivos y negativos para la práctica todos ellos con sus respectivas alternativas de respuesta.
La información fue analizada mediante la utilización de la estadística descriptiva, calculando las frecuencias, media aritmética, desviación estándar, Ji Cuadrada, análisis bivariado de Pearson, para 95\% de confianza, con el apoyo de Excel y el software estadístico SPSS v21.0

\section{RESULTADOS.}

\section{Desempeño docente general.}

De los resultados obtenidos de la toma de decisiones podemos afirmar que se encontró que el desempeño docente a criterio de los estudiantes de enfermería estuvo comprendido entre 46,5 a 66 puntos con una media de 58,73 $\pm 3,23$ (excelente) comparado con la marca de clase (límite inferior + límite superior/2) se encontró una diferencia de 3,23 IC95,0\%=[1,6778 - 4,7958]; por lo que con un $\mathrm{p}$-valor $=0,000$ podemos concluir que la evaluación del desempeño docente en la práctica de la asignatura de Salud del Adulto y Anciano es Excelente (Tabla $\mathrm{N}^{\circ} 1$ ).

Tabla $N^{\circ}$ 1: Desempeño docente a percepción de los estudiantes de enfermería sobre la enseñanza en la práctica clínica de la asignatura Enfermería en Salud del Adulto y Anciano, 2017

\begin{tabular}{|c|c|c|c|c|c|c|c|c|c|c|}
\hline \multicolumn{5}{|c|}{ Estadística descriptiva } & \multicolumn{3}{|c|}{ Diferencia } & \multirow{2}{*}{\multicolumn{3}{|c|}{$\begin{array}{c}\text { Prueba } \\
\text { estadística }\end{array}$}} \\
\hline & & servado & & Esperado & & IC & $5,0 \%$ & & & \\
\hline Media & D.S & Mínimo & Máximo & Media & $\bar{x}$ & Mínimo & Máximo & $\mathrm{T}$ & $\mathrm{gl}$ & p-valor \\
\hline 58,73 & 4,7 & 46,50 & 66,0 & 55,5 & 3,23 & 1,6778 & 4,7958 & 4,207 & 37 & 0,000 \\
\hline
\end{tabular}

\section{Desempeño docente por áreas.}

De los resultados obtenidos de la toma de decisiones podemos afirmar que se reportó que el desempeño docente a criterio de los estudiantes de enfermería en el área de Medicina estuvo comprendido entre 40,0 (Bueno) a 66 puntos con una media de 56,05 $\pm 5,3$ comparado con la marca

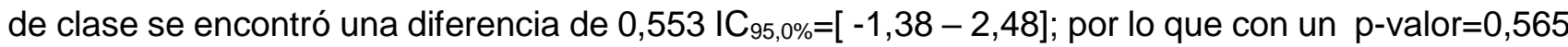
podemos concluir que la evaluación del desempeño docente en la práctica de la asignatura de Salud del Adulto y Anciano en el área de medicina es de Bueno a Excelente (Tabla $N^{\circ} 2$ ).

Tabla $N^{\circ}$ 2: Desempeño docente en el área de Medicina a percepción de los estudiantes de enfermería sobre la enseñanza en la práctica clínica de la asignatura Enfermería en Salud del Adulto y Anciano, 2017

\begin{tabular}{|c|c|c|c|c|c|c|c|c|c|c|}
\hline \multicolumn{5}{|c|}{ Estadística descriptiva } & \multicolumn{3}{|c|}{ Diferencia } & \multirow{2}{*}{\multicolumn{3}{|c|}{$\begin{array}{c}\text { Prueba } \\
\text { estadística }\end{array}$}} \\
\hline \multicolumn{4}{|c|}{ Observado } & \multirow{2}{*}{$\begin{array}{c}\text { Esperado } \\
\text { Media }\end{array}$} & \multicolumn{3}{|c|}{ IC $95,0 \%$} & & & \\
\hline Media & D.S & Mínimo & Máximo & & $\bar{x}$ & Mínimo & Máximo & $\mathrm{T}$ & gl & $\mathrm{p}$-valor \\
\hline 56,05 & 5,8 & 40,0 & 66,0 & 55,5 & 0,553 & $-1,38$ & 2,48 & 0,580 & 37 & 0,565 \\
\hline
\end{tabular}


Se encontró que el desempeño docente a criterio de los estudiantes de enfermería en el área de Cirugía estuvo comprendido entre 45,0 a 66,0 puntos con una media de $58,84 \pm 5,5$; comparado con la marca de clase se encontró una diferencia de $3,342 \mathrm{IC}_{95,0 \%}=[1,52-5,16]$; por lo que con un $p$-valor $=0,001$ podemos concluir que la evaluación del desempeño docente en la práctica de la asignatura de Salud del Adulto y Anciano en el área de Cirugía es excelente (Tabla $N^{\circ} 3$ ).

Tabla N 3: Desempeño docente en el área de Cirugía a percepción de los estudiantes de enfermería sobre la enseñanza en la práctica clínica de la asignatura Enfermería en Salud del Adulto y Anciano, 2017

\begin{tabular}{|c|c|c|c|c|c|c|c|c|c|c|}
\hline \multicolumn{5}{|c|}{ Estadística descriptiva } & \multirow{2}{*}{\multicolumn{3}{|c|}{$\begin{array}{l}\text { Diferencia } \\
\text { IC } 95,0 \%\end{array}$}} & \multirow{2}{*}{\multicolumn{3}{|c|}{$\begin{array}{c}\text { Prueba } \\
\text { estadística }\end{array}$}} \\
\hline & & servado & & Esperado & & & & & & \\
\hline Media & D.S & Mínimo & Máximo & Media & $\bar{x}$ & Mínimo & Máximo & $\mathrm{T}$ & $\mathrm{gl}$ & $p$-valor \\
\hline 58,84 & 5,5 & 45,0 & 66,0 & 55,5 & 3,342 & 1,52 & 5,16 & 3,723 & 37 & 0,001 \\
\hline
\end{tabular}

Se halló que el desempeño docente a criterio de los estudiantes de enfermería en sala de operaciones estuvo comprendido entre 50,0 a 66,0 puntos con una media de $59,74 \pm 4,9$; comparado con la marca de clase se encontró una diferencia de $4,237 I_{95,0 \%}=[2,60-5,88]$; por lo que con un $\quad$-valor $=0,000$ podemos concluir que la evaluación del desempeño docente en la práctica de la asignatura de Salud del Adulto y Anciano en sala de operaciones es excelente (Tabla $\mathrm{N}^{\circ} 4$ ).

Tabla $N^{\circ}$ 4: Desempeño docente en sala de operaciones a percepción de los estudiantes de enfermería sobre la enseñanza en la práctica clínica de la asignatura Enfermería en Salud del Adulto y Anciano, 2017

\begin{tabular}{|c|c|c|c|c|c|c|c|c|c|c|}
\hline \multicolumn{5}{|c|}{ Estadística descriptiva } & \multicolumn{3}{|c|}{ Diferencia } & \multirow{2}{*}{\multicolumn{3}{|c|}{$\begin{array}{c}\text { Prueba } \\
\text { estadística }\end{array}$}} \\
\hline & & servado & & Esperado & & IC & $5,0 \%$ & & & \\
\hline Media & D.S & Mínimo & Máximo & Media & $\bar{x}$ & Mínimo & Máximo & $\mathrm{T}$ & gl & p-valor \\
\hline 59,74 & 4,9 & 50,0 & 66,0 & 55,5 & 4,237 & 2,60 & 5,88 & 5,240 & 37 & 0,000 \\
\hline
\end{tabular}

Se reportó que el desempeño docente a criterio de los estudiantes en sala de operaciones estuvo comprendido entre 46,0 a 66,0 puntos con una media de $60,32 \pm 5,0$; comparado con la marca de clase se encontró una diferencia de 4,816 IC $\mathrm{IS}, 0 \%_{\text {= }}=[3,15-6,48]$; por lo que con un $p$-valor $=0,000$ podemos concluir que la evaluación del desempeño docente en la práctica de la asignatura de Salud del Adulto y Anciano en Recuperación Postoperatorio es excelente (Tabla $N^{\circ} 5$ ).

Tabla N 5: Desempeño docente en Recuperación Postoperatorio a percepción de los estudiantes de enfermería sobre la enseñanza en la práctica clínica de la asignatura Enfermería en Salud del Adulto y Anciano, 2017

\begin{tabular}{|c|c|c|c|c|c|c|c|c|c|c|}
\hline \multicolumn{5}{|c|}{ Estadística descriptiva } & \multicolumn{3}{|c|}{ Diferencia } & \multirow{2}{*}{\multicolumn{3}{|c|}{$\begin{array}{c}\text { Prueba } \\
\text { estadística }\end{array}$}} \\
\hline & & servado & & Esperado & & IC & $5,0 \%$ & & & \\
\hline Media & D.S & Mínimo & Máximo & Media & $\overline{\mathbf{x}}$ & Mínimo & Máximo & $\mathrm{T}$ & gl & p-valor \\
\hline 60,32 & 5,0 & 46,0 & 66,0 & 55,5 & 4,816 & 3,15 & 6,48 & 5,864 & 37 & 0,000 \\
\hline
\end{tabular}

\section{DISCUSIÓN}

En el presente estudio se contó con 38 participantes alumnos que evaluaron el desempeño docente en la asignatura de Salud del adulto y anciano de la Facultad de Enfermería de la Universidad Nacional San Luis Gonzaga de Ica. En ese sentido se encontró que la evaluación del desempeño docente en la práctica de la asignatura de Salud del Adulto y Anciano es Excelente ( $p$ valor $=0,000$ ).

Este resultado concuerda con lo reportado por Meza Lagos et a (2008) (7), en cuyo estudio se concluyó que el $51 \%$ de alumnas de 
Segunda Especialización en Enfermería reportaron satisfacción con la metodología empleada en el proceso de enseñanza aprendizaje. No obstante, estudios como los de Arica V, Garcilazo A. (8) y Martínez M (9), reportaron parámetros de satisfacción que no alcanzaron niveles de excelencia.

En nuestro estudio se halló también que la evaluación del desempeño docente en la práctica de la asignatura de Salud del Adulto y Anciano en el área de medicina es de Bueno a Excelente. $(p=0,565)$, en el área de Cirugía es excelente $(p$-valor $=0,001)$ al igual que en sala de operaciones y en Recuperación post operatoria (con $p=0,000$ para ambas asignaturas). Estos resultados no han encontrado estudios similares con cuyos resultados se pueda evidenciar si existe o no concordancia.

La evaluación del desempeño docente es una estrategia para promover y favorecer el desarrollo del profesorado, que ha de asumirse como una opción de reflexión y de mejora de la realidad, y debe ser entendida adecuadamente para posibilitar el perfeccionamiento de los docentes. A través de la evaluación es posible identificar las cualidades que conforman a un buen profesor para generar políticas académicas que coadyuven a su mejoramiento cualitativo.

\section{CONCLUSIONES}

La evaluación del desempeño docente en la práctica de la asignatura de Salud del Adulto y Anciano en forma global es Excelente. ( $p$ $=0.000$ ). Por áreas en la práctica de la asignatura, la evaluación fue excelente en los cuatro: Medicina $(p=0,565)$, Cirugía $(p=0.001)$, sala de operaciones $(p=0.000)$ y Recuperación Postoperatorio $(P=0.000)$.

\section{RECOMENDACIONES}

Se debe continuar impartiendo la asignatura de Salud del Adulto y anciano en sus diferentes áreas con el mismo ímpetu y esfuerzo como en la actualidad, buscando a su vez promover la educación continua de los docentes que conforman la cátedra respectiva.
Asimismo promover estudios similares empleando también instrumentos que midan niveles de satisfacción o escalas de percepción por ser estos parámetros los más empleados en la evaluación de desempeño docente.

\section{Correspondencia:}

Mg. Rosa Hernández Onofra

Correo electrónico: rosaherono@hotmail.com Celular: 947877737

\section{REFERENCIAS BIBLIOGRÁFICAS.}

1. Rueda M, Luna E. La evaluación del desempeño docente en las universidades públicas de México. Primer coloquio nacional de evaluación del desempeño docente en educación superior. México; 2010. p7-25

2. Muñoz J, Ríos $M$, Abalde E. Evaluación Docente vs Evaluación de la calidad. México. Ed Relieve 2002: 8(002): 103-134.

3. Sánchez S, Domínguez A. Elaboración de un instrumento de viñetas para evaluar el desempeño docente. México. Rev. Mexicana de Investigación Educativa 2008; 13(37): 625-648

4. Díaz W. Formación del profesorado universitario, evaluación de la actividad docente, recursos y promoción. Rev. Estudios Pedagógicos Chile 2016; 42(1): 65-85

5. Centro Interuniversitario de desarrollo CINDA. Evaluación del desempeño docente y calidad de la docencia universitaria. MINEDUC. Santiago de Chile; 2007.

6. Colina Z, Medina N, Parra D, Cedrós J, Montoya C. Modelo para la evaluación del desempeño en la función docencia universitaria. Maracaibo, Venezuela. Rev. Investigación Educativa, 2008; 12(22): 99 $-126$. 
7. Meza L, Pedreschi J. Nivel de satisfacción de las alumnas de la segunda especialización en Enfermería por metodología empleada en el proceso de enseñanza aprendizaje en la UNMSM 2002.Trabajo de Investigación de Especialidad .Lima, Perú. Facultad de Medicina Humana, Escuela de Pos-Grado, Universidad Nacional Mayor de San Marcos; 2008.

8. Arica V, Garcilazo A. Grado de Satisfacción por la enseñanza teórico practico en la especialidad de enfermería intensivista, según opinión de los estudiantes de la UNMSM Abril-Agosto 2005.Tesis Segunda Especialidad .Lima, Perú. Universidad Nacional Mayor de San Marcos; 2005.
9. Martínez M. Encuesta para la evaluación del desempeño docente. Facultad de Ciencias Exactas. Universidad del Centro de la Provincia de Buenos Aires, Argentina;2013

Recibido: $11 / 05 / 2018$

Aprobado para publicación: 28/08/18 